\title{
MOBILITY OF MULTIBODY SYSTEMS IN TERMS OF THEIR INCORRECTNESS
}

The paper presents examples of incorrectness from the point of view of body and Multibody System (MBS) kinematics. This incorrectness should be kept in mind when modeling Virtual Prototypes (VP) of MBS. During the analysis or synthesis of MBS it is necessary to correctly determine the degree of freedom (DOF) of mechanisms in order to properly design the mechanism. The basic relation for the DOF computation does not include parameters such as special dimensions of links or their mutual configuration and geometric constraints. In many cases, these issues require individual approach, i.e. by analyzing the properties of incorrect MBS to identify the causes of their incorrectness and apply the computation method to the actual mobility. The paper contains samples to illustrate a particular topic.

Keywords: MBS, singularity, degrees of freedom, actual mobility, incorrect MBS, kinematics.

\section{Introduction}

The structure and modeling of MBS is a starting and highly important component of theoretical fundamentals. It covers ways of creating kinematic chains, their topology and depiction, classification of geometric constraints and search for a number of freedom (movability), etc.

The DOF of a mechanical system with holonomic geometric constraints is a minimal number of necessary generalized coordinates required to define the system configuration. The DOF has practical use but it must be kept in mind that it has its own limitations. For the modeling of a mechanical system it is necessary to introduce simplifying hypotheses for its computation within the framework of analysis [1]. These hypotheses may cause a significant difference between the model and actual behavior of the mechanism. There is, for instance, a large class of mechanisms (referred to as overconstrained mechanisms) whose mobility is the function of precise dimensions of their parts, chosen geometric constraints or configuration [2, 3 and 4]. Moreover, under certain (singular) configurations, due to a sudden DOF variation, numerical results of particular software may be unreliable unless appropriate precautions are taken $[5$, 6 and 7]. The paper presents some chosen samples of MBS focusing on the computation of incorrectness, which means that DOF is computed according to the commonly used relation not in compliance with the real situation.

\section{The basic concepts}

\subsection{Mobility}

The mobility $n$ of MBS is the number of prescribed position coordinates of input driving links required to uniquely determine the position of output driven links [8]. If the actual mobility $n_{s}$ is identical to the theoretical mobility $n$, i.e. $n_{s}=n$, then we can say that MBS is correct. In the correct MBS, each geometric constraint of class $t$ takes just $t$ degrees of freedom of movement. Position and mobility of bodies in the correct MBS is determined according to the Grubler criterion:

$n=n_{v}(u-1)-\sum_{t=1}^{n_{v}-1} t \cdot s_{t}$,

which satisfies the condition:

$n=n_{s}$,

where $u$ is a total number of links in the MBS, frame included, $n_{v}(u$ - 1) is the mobility of a group of free bodies, $\sum_{t=1}^{n_{v}-1} t s_{t}$ are removed degrees of freedom between pairs of links of the MBS.

The below mentioned relation provides correctness between pairs of links of the MBS:

$S_{t}=\sum_{v=2}^{v_{m}} S_{t v}(v-1)$

\footnotetext{
* 'Alzbeta Sapietova, ${ }^{1}$ Milan Saga, ${ }^{2}$ Alexandr Shimanovsky, ${ }^{1}$ Milan Sapieta

${ }^{1}$ Department of Applied Mechanics, Faculty of Engineering, University of Zilina, Slovakia, E-mail: alzbeta.sapietova@fstroj.uniza.sk

${ }^{2}$ Department of Technical Physics and Engineering Mechanics, Belarusian State University of Transport, Belarus
} 
where $s$ is a number of joints of klass $t$ of all pairs linked in the MBS, $n_{v}(u-1)$ is a number of joints of klass $t$ connecting number $v$ of links, $v$ is a number of bodies linked in $s_{t}$ bodies, $v_{m}$ is a maximum number of bodies in joints of class $t$ in the MBS.

The MBS is considered incorrect if its actual mobility $n_{s}$ differs from the theoretical mobility $n$ determined according to equation 1 ; then it holds:

$n_{s} \neq n$.

All the reasons which cause this condition in MBS are referred to as singularities. Equation 1 does not reflect the real mobility in its entirety for each MBS because it does not contain information either about proportions (dimensions) or about mutual position (configurations) of links and geometrical joints [8].

If $n_{n}$ is used to denote the number of unremoved DOF, the incorrect MBS will have the actual mobility when DOF computed in compliance with the Grübler criterion and the unremoved DOF are summed up:

$n_{s}=n+n_{n}$

Theoretical mobility $n$ of an incorrect MBS may be zero. It can indicate a configuration of links in the MBS or, in the case of negative indication, it can point to an indefinite, unclear structure which still might be real [8]. In spite of it the actual mobility can be $n_{s} \geq 1$. It is due to already mentioned configuration of links and geometric constraints as well as special dimensions.

\subsection{Locked MBS - four bar mechanism}

From the point of view of mobility of bodies and MBS, jam is a situation when the body has one degree of freedom and yet it cannot move. It is caused by the geometry of the body, by mutual configuration of links in the MBS and by force effects [9, 10 and 11]. If redundant constraints in the MBS become inconsistent with other constraints (due to manufacturing differences in length or links or deviation from geometric constraint position), the MBS may jam [8]. There is a four-bar in Fig. 1.

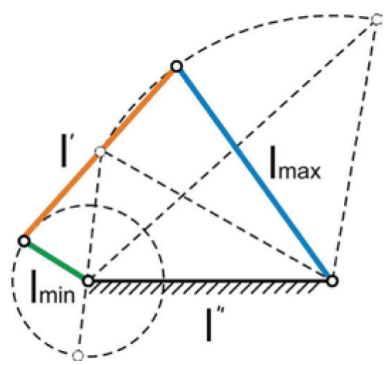

Fig. 1 Kinematic scheme of a four-bar mechanism
If we want the links of a four-bar mechanism in Fig. 1 to perform a rotational motion of $360^{\circ}$, i.e. to function as a crank mechanism, the following relation must hold:

$l_{\min }+l_{\max }=l^{\prime}+l^{\prime \prime}$

where $l_{\min }$ and $l_{\max }$ are lengths of the shortest and the longest link. Then $l^{\prime}$ and $l^{\prime \prime}$ are lengths of the other links. Depending on which of the links is fixed, a crank mechanism, crank-rocker mechanism or rocker mechanism are formed.

\subsection{Instantaneous and permanent singular state of MBS}

- Instantaneous singular state occurs when the links of MBS can displace with infinitely small values of position coordinates (Fig. 2a).

- Permanent singular state occurs when the links of MBS can displace with finite values of position coordinates (Fig. 2b).

Fig. 2a shows a MBS whose links are in a horizontal position and loaded with forces. We get a matrix of system A from the set of static analysis equations. The solvability of the linear system of equation is based on the condition that the system has only one solution if the determinant of the matrix system A is different from zero. From the suggested solution it follows that determinant of the system $D=0$. Then, in the case of configuration of links in Fig. 2a the infinitely small displacement of point A occurs in the horizontal direction. The intersection of the tangents of trajectories of point A from individual links is in infinity. This leads to the virtual displacement in the direction of the tangent resulting into a singular state of MBS. It should be noted that infinitely large forces theoretically arise in the links. In fact, due to clearance in the pins the position of the links changes; but even so, axial forces are very large [12 and 13].
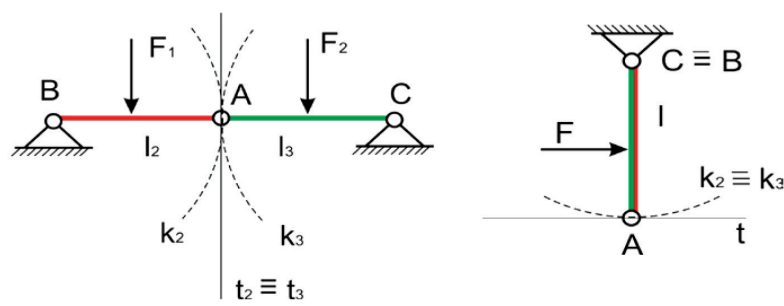

Fig. 2 Three members MBS a) virtual displacement of point $A$, b) final displacement of point $A$

The computation of the DOF according to Grübler: $n=3$. (3-1)-2.3 $=0$. But there is actually a virtual-rotation of links in the system, i.e. $n_{S}=1$. 
$D=\left|\begin{array}{cccccc}1 & 0 & -1 & 0 & 0 & 0 \\ 0 & 1 & 0 & 1 & 0 & 0 \\ 0 & 0 & 0 & l_{2} & 0 & 0 \\ 0 & 0 & 1 & 0 & -1 & 0 \\ 0 & 0 & 0 & -1 & 0 & 1 \\ 0 & 0 & 0 & l_{3} & 0 & 0\end{array}\right|=1 .(-1)^{2}$

$\left|\begin{array}{ccccc}1 & 0 & 1 & 0 & 0 \\ 0 & 0 & l_{2} & 0 & 0 \\ 0 & 1 & 0 & -1 & 0 \\ 0 & 0 & -1 & 0 & 1 \\ 0 & 0 & l_{3} & 0 & 0\end{array}\right|=1 .(-1)^{2} \cdot\left|\begin{array}{cccc}0 & l_{2} & 0 & 0 \\ 1 & 0 & -1 & 0 \\ 0 & -1 & 0 & 1 \\ 0 & l_{3} & 0 & 0\end{array}\right|=1 .(-1)^{3}$.

$$
\left|\begin{array}{ccc}
l_{2} & 0 & 0 \\
-1 & 0 & 1 \\
l_{3} & 0 & 0
\end{array}\right|=0
$$

In Fig. 2b, the links are so oriented that the trajectories of their common point A are identical. In this case, there is a finalrotation of links and the system behaves like a pendulum. The solution of DOF and systems of linear equations is identical to the system in Fig. 2a. In this case a singular mode also occurs because. $n \neq n_{s}$

\section{Incorrect MBS with passive joints (Overconstrained MBS)}

The incorrect MBS is also such a system that contains geometric constraints which do not affect its mobility. This means that if we remove the elements of joints or the entire joint and the mobility of the output link does not change, the joint is totally or partially passive [4].

Total passivity - geometric constraint is from kinematic point of view totally passive and then redundant when after its removal, the actual mobility $n_{S}$ of MBS does not change. Then, it holds that the number of unremoved DOF is equal to the class of constraints $n_{n}=t$. Parameter $\mathrm{t}$ is the number of DOF which the constrain removes. Then it holds:

$n \leq 0$ and $n_{s} \geq 1$

Partial passivity - geometric constraint of a class $t$ is partially passive if it holds that the number of DOF removed by the MBS is smaller than its class, i.e. $n_{o}<\mathrm{t}$ [8].

An example of a totally passive bond is one rotational constraint (from two) to attach casement 2 (Fig. 3). The task is to analyze and compute the mobility of casement attached to the frame with two spatial rotational joints (Fig. 3).

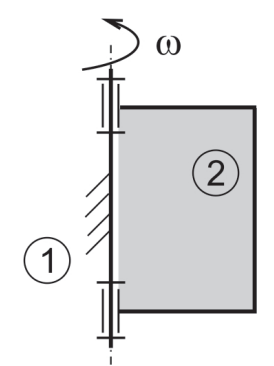

Fig. 3 Kinematic scheme of link with excess joint

We count the DOF according to equation (1):

$n=n_{v}(u-1)-\sum_{t=1}^{n_{v}-1} t . s_{t}=6 .(2-1)-5.2=-4$,

where $S_{t}=2_{52}(2-1)=2$.

Spatial rotational constraint removes 5 DOF. Since, it is excess (redundant), the number of unremoved DOF expresses just the number of DOF which this constraint should remove and it is: $n_{n}=t=5$.

The actual number of DOF of the casement is:

$n_{5}=n+n_{n}=-4+5=1$,

$n \neq n_{5}$.

If we remove the redundant constraint of the body in this example, then the calculation of mobility is according to (1):

$n=n_{v}(u-1)-\sum_{t=1}^{n_{v}-1} t . s_{t}=6 .(2-1)-5.1=1$.

It should also be said that one rotational joint is totally passive in terms of kinematic excess (redundancy). Its task is to achieve favorable conditions for mutual loading of bodies. The casement has always only one DOF regardless of the number of joints attached to the rotating frame. Geometric constraint is totally passive and in geometric terms redundant when, after its removal, the actual mobility $S_{t}$ does not change .

\section{Incorrect MBS with redundant links}

If a link or group of links can be removed and the mobility of output link does not change, the link (group of links) is in excess (redundant) in terms of kinematics, therefore, we remove it before computing the mobility of MBS.

An example of MBS with redundant links is a five-bar system whose links are two and two parallel attached with rotary geometric constraints (Fig. 4). 


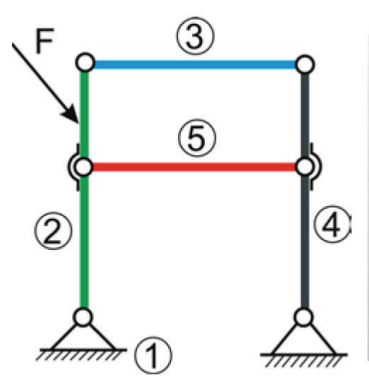

a)

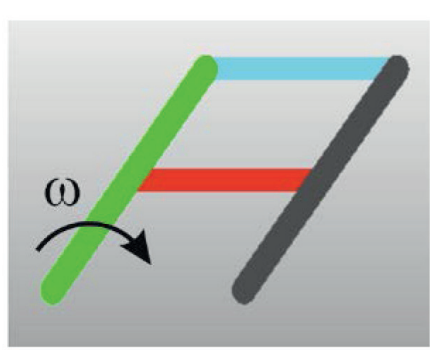

b)
Fig. 4 Kinematic scheme of MBS with excess link

The computation of the DOF will be done as in the previous examples. The number of DOF according to Grubler:

$n=n_{v}(u-1)-\sum_{t=1}^{n_{v}-1} t \cdot s_{t}=3 \cdot(5-1)-2 \cdot 6=0$, $n=0 \neq n_{s}=1$.

After the removal of excess link 5 or 3 , we obtain:

$$
n=n_{v}(u-1)-\sum_{t=1}^{n_{v}-1} t . s_{t}=3 .(4-1)-2.4=1 .
$$

It should also be said that the cause of system mobility is configuration of its links, and the fact is that the links are two plus two parallel links. It is sufficient that one of the links is not parallel and the system will become shape determinate.

\section{Redundant local mobility}

Redundant local mobility is a passive redundant kinematic input which has no influence on the total mobility of the output link of the system.
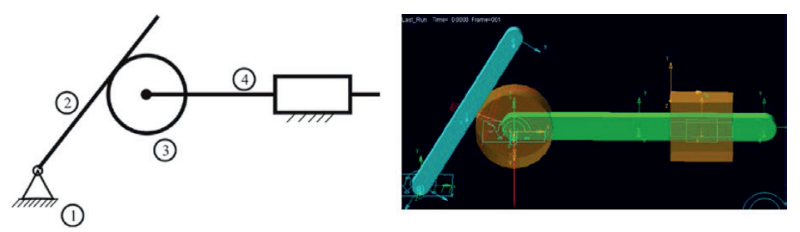

Fig. 5 Kinematic scheme of MBS with excess local mobility

$$
n=n_{v}(u-1)-\sum_{t=1}^{n_{v}-1} t \cdot s_{t}=3 .(4-1)-2.3-1.1=2
$$

If link 4 becomes the frame, link 3 can rotate. From this and also from the computation it follows that the MBS in Fig. 5 has two generalized coordinates. It should be noted that if we investigate the trajectory of an arbitrary point of the working member 2, it does not change even if the input acts on member 3 , i.e. the only driving member will be member 4 .

\section{Freundenstein's criterion of MBS mobility}

To solve MBS mobility which may be both correct and also incorrect, it is possible to use Freundenstein's mobility criterion of planar MBS with global coordinates of the position. It has the form:

$n_{s F}=m-h_{m}$,

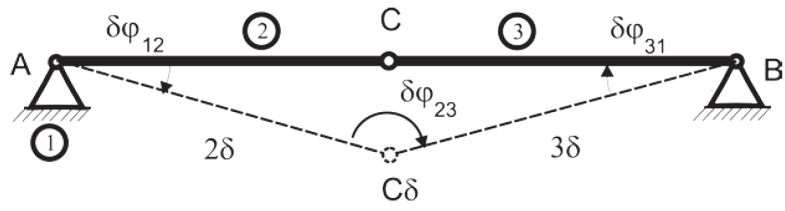

Fig. 6 VMS with marked virtual rotations of links 2 and 3

where $n_{S F}$ is an actual number of DOF of the MBS according to Freundenstein, $m$ is a total number of global coordinates, $h_{m}$ is rank of the Jacobi matrix. Solution of mobility of a planar threebar system of links (Fig. 6) [2] is presented below.

From the kinematic solution we obtain a matrix velocity loop equation for the given system:

$\left[\begin{array}{cc}-h_{2} \sin \varphi_{12} & -h_{3} \sin \varphi_{31} \\ -h_{2} \cos \varphi_{12} & h_{3} \cos \varphi_{31}\end{array}\right]\left[\begin{array}{c}\dot{\varphi}_{12} \\ \dot{\varphi}_{31}\end{array}\right]=\left[\begin{array}{l}0 \\ 0\end{array}\right]$,

then Jacobi matrix:

$\gamma_{d m}=\left[\begin{array}{cc}-h_{2} \sin \varphi_{12} & -h_{3} \sin \varphi_{31} \\ -h_{2} \cos \varphi_{12} & h_{3} \cos \varphi_{31}\end{array}\right]$.

For angles $\varphi_{12}$ and $\varphi_{31}$ following holds:

$\varphi_{12}=\varphi_{31}=0$ and $\varphi_{31}=0 \Rightarrow \gamma_{d m}=\left[\begin{array}{cc}0 & 0 \\ -h_{2} & h_{3}\end{array}\right]$

with $\gamma_{d m}=0$,and $h_{m}=1$,

then, mobility MBS according to (8):

$n_{F}=m-h_{m}=2-1=1$.

Mutual position (lengths, angles) of links and constraint elements allows to links 2 and 3 virtual rotation of $\delta \varphi_{12}$ and $\delta \varphi_{31}$.

According to Grübler $n=0$ and according to Freundenstein $n=n_{s F}=1$, a virtual displacement (rotation) occurs. If det $\gamma_{d m}=0$, then the MBS with incorrect mutual position is in the singular state.

\section{Location and mobility of MBS with rolling constraints}

From the point of view of reaction effects in rolling constraint the following holds: if we do not consider passive resistance, this 
constraint is of class $t=2$. Rolling constraint has both normal and tangential component of the reaction. It can be said that we have 2 constraint conditions in the kinematic model:

1. Tangential point with the frame is also pole $\mathrm{P}$ of relative mobility.

2. The relative velocity at point $\mathrm{P}$ is zero.

So, in terms of kinematics $t=2$.

Rolling constraints can be closed and opened in terms of configuration and shape links. Links may be separated when the constraint is opened. When it is closed, the links cannot be separated.

\subsection{Closed rolling constraint}

Links have the analogue relative position as in the previous solutions (planar three-bar system of bodies) in the constraint with a rolling closed circular shape. They are in a permanent singular mutual position. The constraint is partially passive and has 1 unremoved DOF $n_{n}=1$ (Fig. 7).

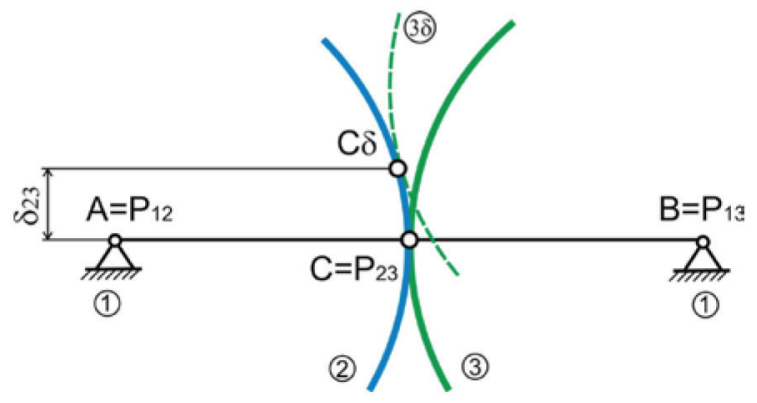

Fig. 7 MBS with rolling closed constraint

An example of the closed constraint is transmission gear. Fig. 8 presents a kinematic scheme of MBS for a switch point machine designed to handle switch points, switch diamonds, and derailers belonging to the equipment for railway operation.
Computation of DOF according to Grubler:

$n=n_{v}(u-1)-\sum_{t=1}^{n_{v}-1} t . S_{1}=$
$n_{v}(u-1)-\sum_{t=1}^{n_{v}-1} t . S_{1}=3 .(5-1)-2.7=-2$.

MBS has 3 types of gear and each of them has one unremoved DOF; then it follows that $n_{n}=3$.

The actual DOF of MBS for switch point machines are:

$n_{s}=n+n_{n}=-2+3=1$.

\subsection{Opened rolling constraint - Incorrectness in terms of structural analysis.}

The opened rolling constraint differs from the closed rolling constraint - in the former, separation of contacting surfaces of bodies may occur. The cause of incorrectness may be dimension and shape of tangential links [11].

In Fig. 9, there is the MBS with open rolling constraint, i.e. a cylinder in a plane. Link 3 is attached to the pin of cylinder 2. To clearly identify the position of the system it is necessary to know the location of the cylinder $p_{13}$ which is the function of rotations of cylinder $\phi_{12}$, and $\phi_{13}$ which is an independent rotation of link 3 towards the frame. This system has 2 DOF. The structural scheme shows that links 1 and 2 are in contact through the rolling constraint, links 2 and 3 are in contact through rotational constraint, and link 3 is not in a direct contact with the frame. The structural scheme (topological model) of MBS does not contain any information about dimensions and mutual configuration of links.
(4)

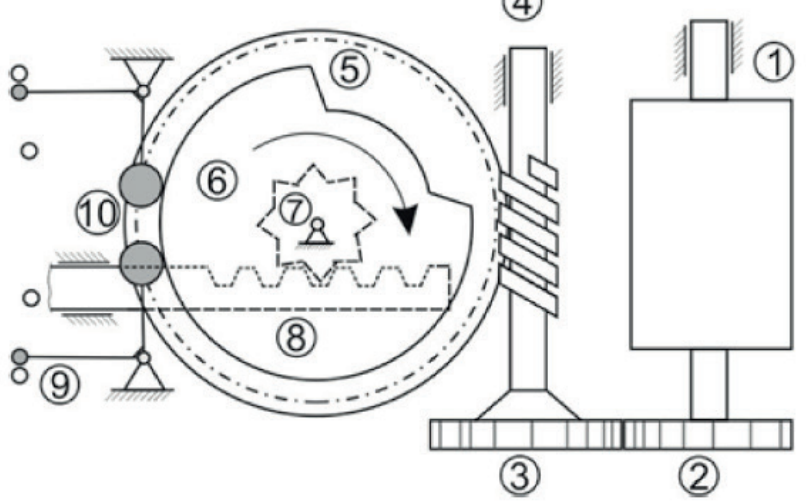

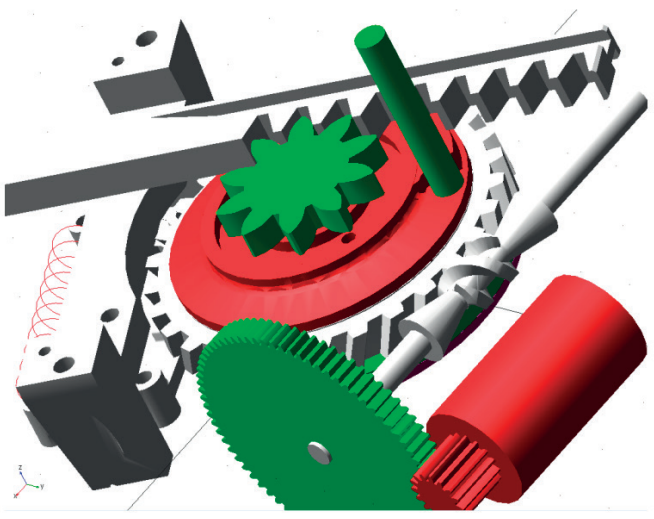

Fig. 8 MBS with mark virtual rotation of links 2 and 3 

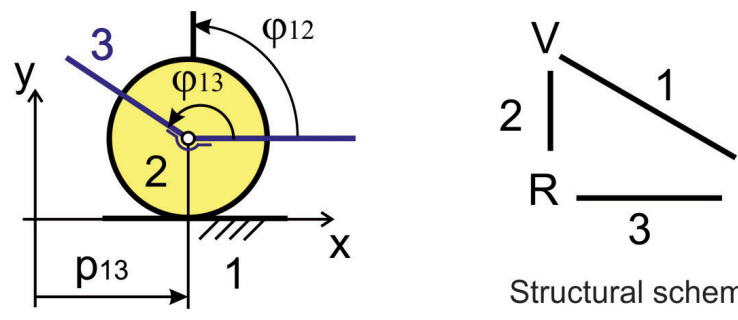

Structural scheme

Fig. 9 MBS with open rolling constraint

Computation according to Grubler shows the actual DOF, but in this case it is incorrectness in terms of structural analysis: $n=n_{v}(u-1)-\sum_{t=1}^{n_{v}-1} t \cdot S_{1}=3 .(3-1)-2.2=2$, $n_{S}=n+n_{n}=2+0=2$.

Parameter $c$ is the total number of local coordinates neighboring (dependent as well as independent) tangential to bodies; i.e. coordinates indicating the relative position (velocity, acceleration) between indicating the relative position (velocity, acceleration) 1,2 and 2, 3, then $\mathrm{c}=2$. Parameter $m$ is the total number of global coordinates, i.e. coordinates between 1, 2 and 1,3 , then $\mathrm{m}=2$.

We need to enter one more global coordinate in order to determine unambiguously the position of the system. It follows that we need 3 joint equations $m_{s}=3$, reflecting the fact that a shape of tangential links is crucial for open roller joints.

Incorrectness in terms of structural analysis:

$c=2, m=2 \neq m_{s}=3$,

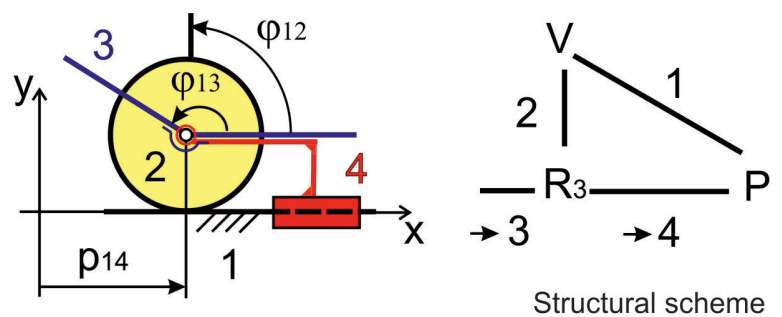

b.)

Fig. 10 Closed rolling constraint compensated by inserting binary link

$n=n_{v}(u-1)-\sum_{t=1}^{n_{v}-1} t \cdot S_{1}=3 \cdot(4-1)$
$-2 .\left(2_{22}(2-1)+1_{23}(3-1)\right) 2=1$,
$n_{S}=n+n_{n}=1+1=2$.

Incorrectness of the open rolling constraint is compensated as we insert a fictional link to the system, which will replace the missing geometric constraint.

This particular incorrectness is compensated by inserting binary link 4 . The centers of osculating circles of links were achieved. Thus the open rolling constraint is transformed to closed, which is permanently partially passive and the structural analysis will be correct (Fig. 10).

\section{Conclusion}

Practical importance of solution of the above mentioned issues lies in the determination of the number of independent degrees in the mechanism to determine a prescribed motion of the output link. This type of analysis can be preliminarily carried out by means of simple equations requiring only the knowledge of the number of links, the number of constraints and nature of their classes. However, they may fail during providing the correct answer.

Here, it is important to realize that it is necessary to orient oneself in basic knowledge which will allow us to:

- recognize and identify the critical configuration of a mechanism,

- compute the DOF of an overconstrained mechanism,

- identify passive or redundant DOF,

- identify the numerical effects due to the DOF variation.

Finally, it can be stated that causes of MBS incorrectness are identified based on analysis of properties of incorrect MBS and computational methods for actual mobility can be applied.

\section{Acknowledgement}

The part of the results of this work has been supported by VEGA grant No. 1/1259/12.

\section{References}

[1] ARNOLD, M., SCHIEHLEN, W.: Simulation Techniques for Applied Dynamics, CISM Courses and Lectures, vol. 507, Italy : Springer Wien : New York, 2008, 313 p., ISBN 978-3-211-89547-4.

[2] FREUDENSTEIN, F., ALIZADE, R.: On the Degree of Freedom of Mechanisms with Variable General Constraint, Proc. of Fourth World Congress on the Theory of Machines and Mechanisms, Newcastle upon Tyne, 1975, vol. I, 51-56. 
[3] PENNESTRI, E., CAVACECE, M., VITA, L.: On the Computation of Degrees-of-freedom: A Didactic Perspective, ASME Paper DETC, 2005, 2005-84109, 1-9.

[4] SAGA, M., ZMINDAK, M., DEKYS, V., SAPIETOVA, A., SEGLA, S.: Selected Methods of Analysis and Synthesis of Mechanical Systems, VTS: University of Zilina, 2009, 360 p., ISBN 978-80-89276-17-2.

[5] KOPAS, P., VASKO, M., HANDRIK, M.: Computational Modeling of the Microplasticization State in the Nodular Cast Iron, Applied Mechanics and Materials, 2014, vol. 474, 285-290, ISSN 16609336.

[6] VASKO, M., GURAN, A., JAKUBOVICOVA, L., KOPAS, P.: Determination the Contact Stress Depending on the Load Rate of the NU220 Roller Bearing, Communications - Scientific Letters of the University of Zilina, vol. 15, No. 2, 2013, 88-94, ISSN 13354205.

[7] SAPIETOVA, A., SAPIETA, M., HYBEN, B.: Sensitivity Analysis Application for Multibody System Synthesis, Applied Mechanics and Materials, vol. 420, 2013, 68-73, Online available since 2013/Sep/27 at www.scientific.net (c (2013) Trans Tech Publications, Switzerland doi:10.4028/www.scientific.net/AMM.420.68 ISSN 1660-9336.

[8] PALCAK, F.: Theory of Mechanisms (in Slovak), $2^{\text {nd }}$ ed., Bratislava : ES SVST, 1993. 166 p., ISBN 80-227-0531-4.

[9] STANCEKOVA, D., SEMCER, J., DERBAS, M., KURNAVA, T.: Methods of Measuring of Residual Stresses and Evaluation of Residual State of Functional Surfaces by x-ray Diffractometric Methods, J. Manufacturing Technology, vol. 13, No. 4, December 2013, 547-552, ISSN 1213-2489.

[10] CZAN, A., TILLOVA, E., SEMCER, J., PILC, J.: Surface and Subsurface Residual Stresses after Machining and their Analysis by x-ray Diffraction, Communications - Scientific Letters of the University of Zilina, vol. 15, No. 2, 2013, 69-76. ISSN 1335-4205.

[11] KUCERA, L., GAJDOSIK, T.: The Vibrodiagnostic of Gears, Proc. of $54^{\text {th }}$ Intern. Conference of Machine Design Departments, September, 2013, Hejnice - Liberec : Technical university, 2013. ISBN 978-80-7372-986-8, 93-98.

[12] KUCERA, L., LUKAC, M., JURAK, L., BRUMERCIK, F.: Hydromechanical Automatic Transmission, Communications Scientific Letters of the University of Zilina, vol. 11, No. 2, 2009, 33-35, ISSN 1335-4205.

[13] LACK, T., GERLICI, J.: Modified Strip Method Utilization for Wheel/Rail Contact Stress Evaluation. 9 $^{\text {th }}$ Intern. Conference on Contact Mechanics and Wear of Rail/wheel Systems (CM 2012), August, 2012, Chengdu : Southwest Jiaotong University, 2012, 87-89. 